\title{
Adaptive Finite Volume Simulation of Electrical Characteristics of Organic Light Emitting Diodes
}

\author{
Yiming $\mathrm{Li}^{1,2}$ and $\mathrm{Pu} \mathrm{Chen}^{2}$ \\ ${ }^{1}$ Department of Computational Nanoelectronics, National Nano Device Laboratories, \\ Hsinchu 300, Taiwan \\ ${ }^{2}$ Microelectronics and Information Systems Research Center, National Chiao Tung University, \\ Hsinchu 300, Taiwan \\ ymliafaculty.nctu.edu.tw
}

\begin{abstract}
In this paper a two-dimensional simulation of organic light emitting devices (OLEDs) using an adaptive computing technique is presented. A set of drift-diffusion equations including models of interface traps is solved numerically to explore the transport property of OLED structures. The adaptive simulation technique is mainly based on the Gummel's decoupling algorithm, a finite volume approximation, a monotone iterative method, a posteriori error estimation, and an unstructured meshing scheme. With this computational approach, we investigate the intrinsic and terminal voltage-current characteristics of OLEDs with respect to different material parameters, thickness of materials, and length of structure.
\end{abstract}

\section{Introduction}

Organic electroluminescence has been of great interest in various display applications. Organic light emitting diode (OLED) displays are lightweight, durable, power efficient and ideal for portable applications [1]. They have lower material costs and fewer processing steps than their liquid crystal display (LCD) counterparts. As such, the OLED display appears to be a strong candidate as a replacement technology in a variety of mobile application areas. OLEDs with different thin-film structures, consisting of emitter and carrier transport layers, have recently been reported [2], [3], [4], [5], [6]. According to the simple device geometry in OLEDs, one-dimensional (1D) transport model, the drift-diffusion model, has generally been solved along the transport direction for studying the electrical properties of OLEDs [4], [5], [6]. However, a multidimensional modeling and simulation plays a crucial role for exploring the effect of device structure and material on the electrical characteristics of OLEDs.

In this paper a set of drift-diffusion (DD) equations is solved with an adaptive computing technique [7], [8], [9], [10] for a two-dimensional (2D) simulation of OLEDs. For the simulation of OLEDs, the DD equations consist of the Poisson equation, the current continuity equation of electron, the current continuity equation of hole, and models of interface traps. First of all we decouple the three partial differential equations (PDEs) according to the Gummel's procedure. Based on adaptive unstructured mesh and finite volume (FV) approximation, each decoupled PDE is discretized and then solved by means of the monotone iterative (MI) method instead of 
Newton's iteration (NI) method. The method of monotone iteration is a constructive alternative for numerical solutions of PDEs. It has been reported that, compared with the NI method, the major features of the MI method are (1) it converges globally with any arbitrary initial guesses; (2) its implementation is much easier than NI method; and (3) it is inherently ready for parallelization [8]. Furthermore, due to the efficient posteriori error estimation, the variation of physical quantities, such as the gradients of potential and current density, can be automatically tracked. Therefore, the terminal characteristics are accurately calculated. The proposed adaptive computing technique shows the simulation accuracy and numerical robustness for the simulation of $2 \mathrm{D}$ OLEDs. Effects of geometry, the trap density and the Schottky barrier height [11] on the current-voltage (I-V) curves of the simulated 2D OLED are examined using the developed 2D simulation program.

This paper is organized as follows. In the section 2, we state the transport model and the adaptive computing technique for the 2D simulation of OLED. In the section 3 , the results of numerical simulation are discussed. Finally we draw the conclusions.

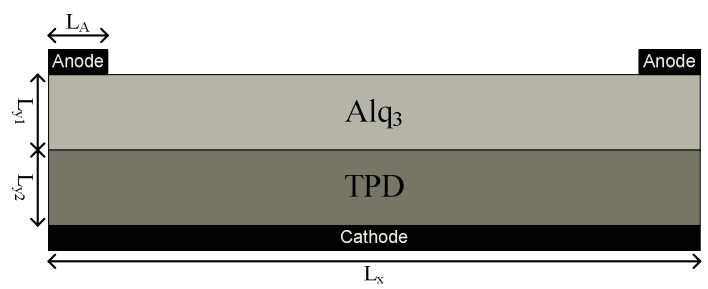

Fig. 1. A cross-sectional view of the studied OLED structure, where $L_{A}$ is the length of contacts of anode and $\mathrm{L}_{\mathrm{x}}$ is the length of contact of cathode. The $\mathrm{L}_{\mathrm{y} 1}$ is the width of material Alq3 which is the layer of electron transport and the $\mathrm{L}_{\mathrm{y} 2}$ is the width of material TPD which is the layer of hole transport

\section{Mathematical Model and Computational Methodology}

Based on the well-known inorganic charge transport continuum model [9], the driftdiffusion model, electron and hole transport in the OLED is described using the current continuity equations coupled to the Poisson equation [11]. Along with the appropriate boundary conditions, which for OLEDs require appropriate formalisms for current injection at each of the contacts, these equations are solved to obtain solutions for the electrostatic potential, electric field, carrier densities, and current densities for electron and hole, respectively. The investigated 2D structure of OLED, shown in Fig. 1 , is a TPD/Alq ${ }_{3}$ two-layer device. Modeling of traps in numerical simulation of OLEDs is widely debated [3]. The electron-hole mobility taking the field-dependent Poole-Frenkel form in the current continuity equations of electron and hole is for shallow trapping of carriers. For deep traps, an extra recombination term and inclusion of their charge in the Poisson equation are considered. Deep traps can be described by a discrete and exponential distribution. The simulated OLED is based on the 2D structure of the tris-(8-hydoroxyquinoline)-aluminum $\left(\mathrm{Alq}_{3}\right)$ for the layer of electron transport and the triphenyl-diamine (TPD) for the layer of hole transport. As 
shown in Fig. 1, we assume the length of anode $\left(\mathrm{L}_{\mathrm{A}}\right)$ is equal to $20 \mathrm{~nm}$, the length of cathode $\left(\mathrm{L}_{\mathrm{X}}\right)$ is equal to $400 \mathrm{~nm}$, and the thicknesses of $\mathrm{Alq}_{3}$ layer $\left(\mathrm{L}_{\mathrm{y} 1}\right)$ and TPD layer $\left(\mathrm{L}_{\mathrm{y} 2}\right)$ are equal to $40 \mathrm{~nm}$, respectively. We solve the steady-state DD model [1114], which consists of

$$
\begin{gathered}
\Delta \psi=-\frac{q}{\varepsilon}\left(p-n-N_{A}+N_{D}-n_{t}+p_{t}\right), \\
\nabla J_{n}=-q(G-R), \text { and } \\
\nabla J_{p}=-q(G-R) .
\end{gathered}
$$

The current equations, shown in Eqs. (2) and (3), for electron and hole are given by

$$
\begin{aligned}
& J_{n}=-q \mu_{n}\left(n E-\frac{K_{B} T}{q} \nabla n\right) \text { and } \\
& J_{p}=-q \mu_{p}\left(p E+\frac{K_{B} T}{q} \nabla p\right) .
\end{aligned}
$$

In Eq. (1), $\psi$ is the electrostatic potential, $\varepsilon$ is the dielectric constant, $N_{A}$ and $N_{D}$ are the densities of acceptor and donor, $n_{t}$ and $p_{t}$ are the densities of trapped electrons and holes, respectively. Maxwell-Boltzmann statistics is adopted for the electron and hole densities. In Eqs. (2) and (3) $G$ is the electron and hole generation rate and the carrier generation by thermal excitation across the gap is assumed. Two carrier recombination rates, the optical recombination rate $R_{\text {opt }}$ and the Shockley-Read-Hall recombination rate $R_{s r h}$ are assumed in the simulation [11]. We consider here the densities of trapped electrons and holes for the $j^{\text {th }}$ trap level

$$
n_{t}=\frac{N_{t j}}{1+\frac{1}{g} e^{\frac{E_{t j}-E_{f n}}{K_{B} T}}} \text { and } p_{t}=\frac{P_{t j}}{1+\frac{1}{g} e^{-\frac{E_{t j}-E_{f p}}{K_{B} T}}},
$$

where $N_{t j}\left(P_{t j}\right)$ is the electron (hole) trap density, $E_{t j}$ is the trap energy relative to the conduction band edge, $\mathrm{g}$ is the trap degeneracy, and $E_{f n}\left(E_{f p}\right)$ is the electron (hole) quasi-Fermi level. Boundary conditions are assumed for the DD model above [11-14].

In the solution procedure, the adaptive computing is mainly based on the Gummel's decoupling algorithm, the FV approximation, the MI method, a posteriori error estimation, and an unstructured meshing technique. This simulation methodology has been developed in our recent work for semiconductor device simulation [7], [8], [9], [10]. Each Gummel decoupled PDE is approximated with the FV method over unstructured meshes. The corresponding system of nonlinear algebraic equations of the FV approximated PDE is solved with the MI method and the posteriori error estimation scheme is applied to assess the quality of computed solutions. It has been shown that the method of MI converges monotonically [8]. The adaptive mechanism is based on an estimation of the gradient of computed solutions, such as the electrostatic potential, the carrier density, and the current density. A posteriori error estimation is 
applied to provide local error indicators for incorporation into the mesh refinement strategy. The local error indicators guide the adaptive refinement process.

\section{Results and Discussion}

In this section we first present the computational efficiency of the method by solving the OLED under a given biasing condition. The applied anode voltage and cathode voltage on the OLED are $10 \mathrm{~V}$ and $0 \mathrm{~V}$, respectively, and the barrier height on the contact is assumed to be $0.3 \mathrm{eV}$. For this testing case, the obtained initial and final refined meshes are shown in Fig. 2. The stopping criteria for the MI and Gummel's loops are $10^{-6}$ and $10^{-3}$, respectively. The initial mesh has 153 nodes and the final one consists of 1681 nodes. The adaptive computing process includes 5 refinement levels, which shows the computational efficiency. Different simulation cases are further performed to explore the intrinsic and terminal electrical characteristics of the OLEDs.

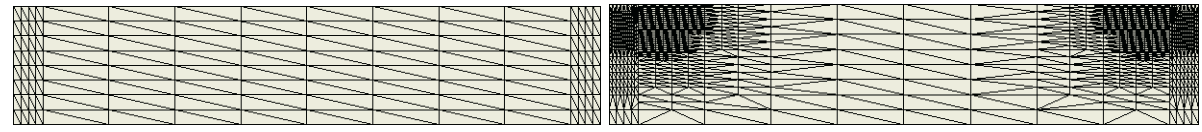

Fig. 2. The left figure is the initial mesh and the right one the $5^{\text {th }}$ refined final mesh
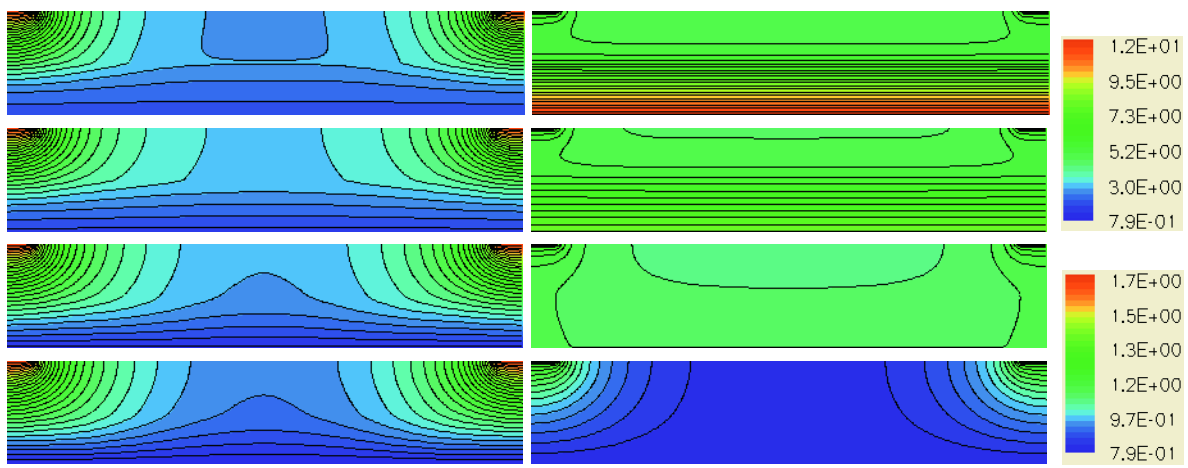

Fig. 3. The simulated potential of the OLED under the $10 \mathrm{~V}$ (left column) and the zero bias (right one). The barrier height is $0.3 \mathrm{eV}$ (top figure), $0.6 \mathrm{eV}, 0.9 \mathrm{eV}$, and $1.2 \mathrm{eV}$ (bottom one)

We note that the simulation of OLEDs require the input of accurate material parameters, such as density of state, barrier height, and carrier mobility. However, unlike the case for inorganic semiconductor devices, these material parameters are often poorly characterized and are strongly dependent upon the fabricated samples. Our selection of the material parameters provides only the starting point for the electrical simulation of OLEDs. Good agreement between simulation and measurement should be subject to further calibration. Contour plots of the electrostatic potential and the electron density are shown in Figs. 3 and 4, respectively, where the OLED under two different biasing conditions the anode voltage is equal to $10.0 \mathrm{~V}$ and $0 \mathrm{~V}$, are simulated. The cathode voltage is fixed at $0.0 \mathrm{~V}$ and the barrier height of contact varies from $0.3 \mathrm{eV}$ to $1.2 \mathrm{eV}$ 
with step $0.3 \mathrm{eV}$. The unit of the color bars, shown in Fig. 3, is in Volt. The computed electrostatic potential significantly shows the importance of selection of barrier height. Different barrier heights imply different current injection. The unit of the color bars of electron density is with per meter cubic. Figures 3 and 4 demonstrate the necessary for the advanced design of OLED structures by using a 2D simulation.
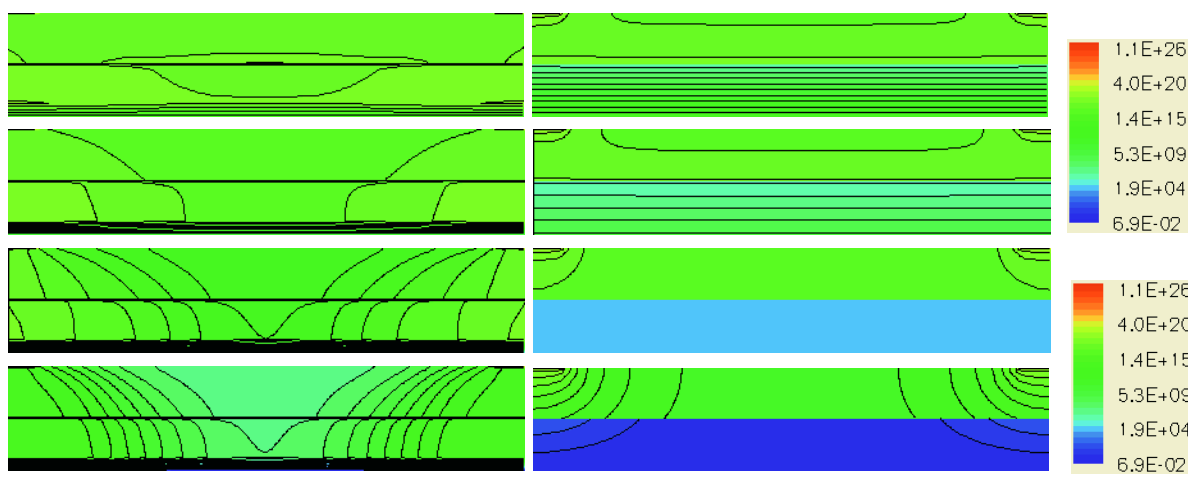

Fig. 4. The contour plots of the simulated electron density. The setting is the same with Fig. 3

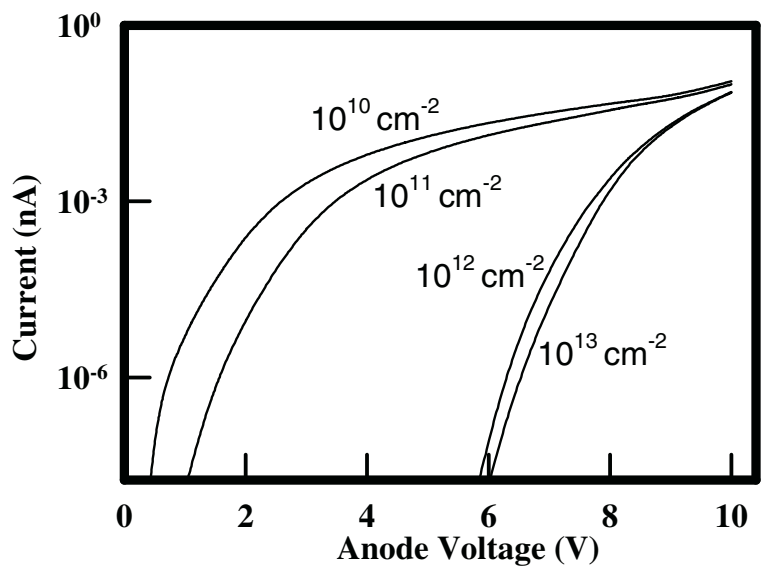

Fig. 5. The I-V curves of the simulated OLED with different trap densities of electron

To explore the effect of trap density on the transport characteristics, we simulate the current-voltage (I-V) curves with respect to different electron trap densities, where the hole trap density is neglected. As shown in Fig. 5, the I-V curves per unit area are calculated with different densities of traps which range from $10^{10} \mathrm{~cm}^{-2}$ to $10^{13} \mathrm{~cm}^{-2}$. The anode voltage is equal to $10 \mathrm{~V}$ and the barrier height is fixed at $0.3 \mathrm{eV}$. It is found that the higher electron trap densities get no significant benefit to improve the electrical performance of the OLED. The choice of OLED's contacts is crucial, and it af- 
fects the minority and majority currents, the recombination rates, and the efficiency. At the interface of metal and semiconductor of OLED, the variation of barrier heights is wide. The operation of OLED depends upon the asymmetry of the barrier heights at the two contacts, shown in Fig. 1; ITO is one of the preferred anode materials due to the transparency and relatively high work function. Metal, such as $\mathrm{Al}, \mathrm{Ca}, \mathrm{or} \mathrm{Mg}$, with low work functions is selected as cathode material. To explore the effect of the Schottky barrier height on the I-V relationships [11], the Schottky barrier height on the contacts is simply varied from 0.0 to $1.2 \mathrm{eV}$, shown in Fig. 6.

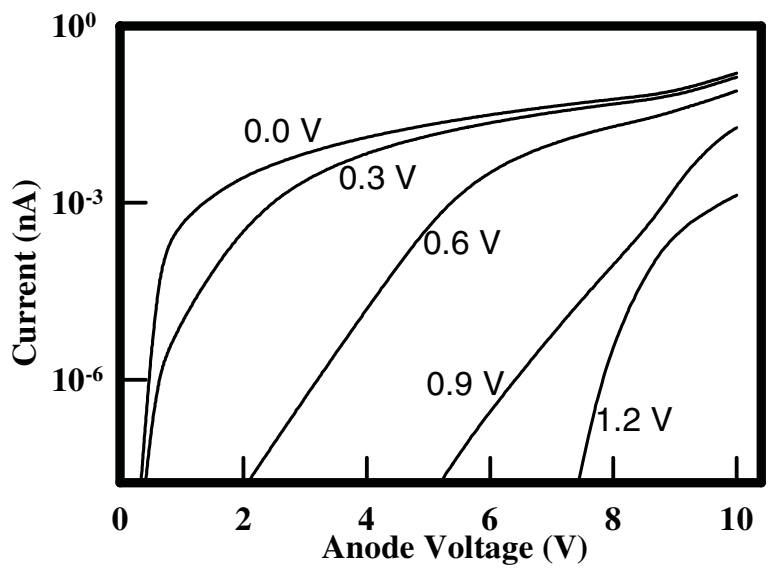

Fig. 6. The I-V curves of the simulated OLED with different Schottky barrier heights

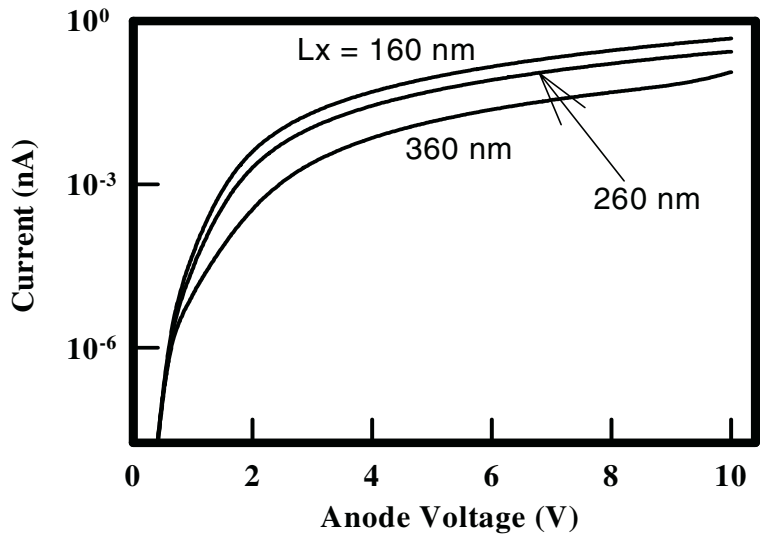

Fig. 7. The I-V curves of the simulated OLED with different lateral length $L_{x}$ 
With the 2D simulation, the lateral length and thickness of transport layer are changed for exploring the I-V curves. It is found that small $\mathrm{L}_{\mathrm{x}}$ produces high current level when the $\mathrm{L}_{\mathrm{y} 1}$ and $\mathrm{L}_{\mathrm{y} 2}$ are fixed, shown in Fig. 7. Due to uniform distribution of electric fields, the small variation on $\mathrm{L}_{\mathrm{y} 1}$ and $\mathrm{L}_{\mathrm{y} 2}$ does not significantly alter the level of current.

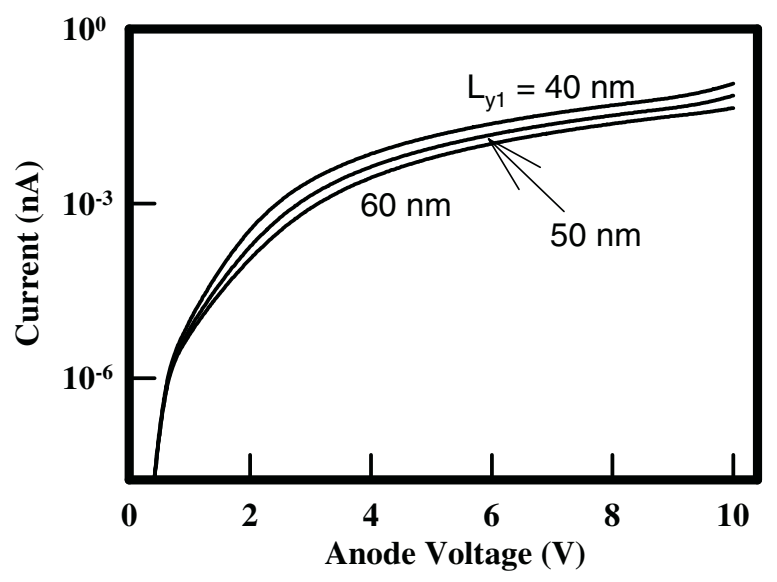

Fig. 8. The I-V curves with respect to different thicknesses of electron transport layer $L_{y 1}$

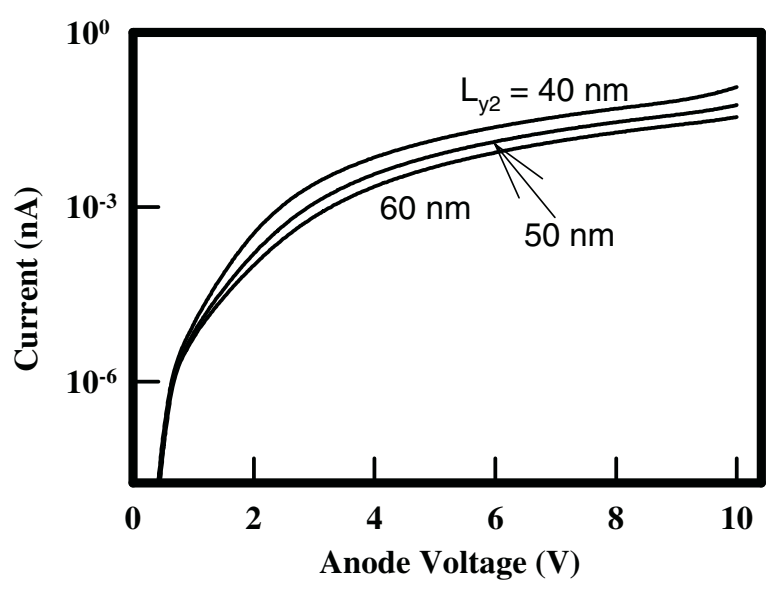

Fig. 9. The I-V curves with respect to different thicknesses of hole transport layer $\mathrm{L}_{\mathrm{y} 2}$ 


\section{Conclusions}

In this paper we have successfully applied adaptive computing technique to solve the 2D DD model for the simulation of electrical characteristics of OLEDs. Effects of the carrier traps and device geometry on the transport properties have been studied. The 2D macroscopic modeling and simulation is a starting point for theoretical investigation of electrical characteristics of OLEDs. It benefits the design of structure and optimization of characteristics. Application of technology computer-aided design software to modern display industry requires accurate modeling and calibration of material parameters. In contrast to the macroscopic simulation, we believe that microscopic approaches will physically provide accurate calculation.

\section{Acknowledgments}

This work is supported in part by the National Science Council (NSC) of TAIWAN under contracts NSC-93-2215-E-429-008 and NSC 93-2752-E-009-002-PAE, and the grant of the Ministry of Economic Affairs, Taiwan under contracts 92-EC-17-A-07S1-0011 and 93-EC-17-A-07-S1-0011.

\section{References}

1. Goh, J.-C., Chung, H.-J., Jang, J., Han, C.-H.: A New Pixel Circuit for Active Matrix Organic Light Emitting Diodes. IEEE Elec. Dev. Lett. 23 (2002) 544-546

2. Ruhstaller, B. Carter, S.A., Barth, S., Riel, H., Riess, W., Scott J. C.: Transient and SteadyState Behavior of Space Charges in Multilayer Organic Light-Emitting Diodes. J. Appl. Phys. 89 (2001) 4575-4586

3. Waler, A. B., Kambili A., Martin S. J.: Electrical Transport Modelling in Organic Electroluminescent Devices. J. Phys.: Condens. Matter 14 (2002) 9825-9876

4. Ruhstaller, B., Beierlein, T., Riel, H., Karg, S., Scott, J. C., Riess W.: Simulating Electronic and Optical Processes in Multilayer Organic Light-Emitting Devices. IEEE J. Sel. Topics Quantum Elec. 9 (2003) 723-731

5. Blades, C. D. J., Walker, A. B.: Simulation of Organic Light Emitting Diodes. Synth. Met. 111-112 (2000) 335-340

6. Barth, S., Müller, P., Riel, H., Seidler, P. F., Riess, W., Vestweber, H., Bässler, H.: Electron Mobility in Alq Thin Films Determined via Transient Electroluminescence from Single- and Multilayer Organic Light-Emitting Diodes. J. Appl. Phys. 89 (2001) 3711

7. Li, Y., Yu, S.-M.: A Two-Dimensional Quantum Transport Simulation of Nanoscale Double-Gate MOSFETs using Parallel Adaptive Technique. IEICE Trans. Info. \& Sys. E87-D (2004) 1751-1758

8. Li, Y.: A Parallel Monotone Iterative Method for the Numerical Solution of Multidimensional Semiconductor Poisson Equation. Comput. Phys. Commun. 153 (2003) 359-372

9. Li, Y., Sze, S, M., Chao, T.-S.: A Practical Implementation of Parallel Dynamic Load Balancing for Adaptive Computing in VLSI Device Simulation. Eng. Comput. 18 (2002) 124-137 
10. Li, Y., Liu, J.-L., Chao, T.-S., Sze, S. M.: A New Parallel Adaptive Finite Volume Method for the Numerical Simulation of Semiconductor Devices. Comput. Phys. Commun. 142 (2001) 285-289

11. Sze, S. M.: Physics of semiconductor devices. Wiley-Interscience, New York (1981)

12. Crone, B. K., Davids. P. S., Campbell. I. H., Smith, D. L.: Device Model Investigation of Bilayer Organic Light Emitting Diodes. J. Appl. Phys. 87 (2000) 1974-1982

13. Lupton, J. M., Samuel, I. D. W.: Temperature-Dependent Single Carrier Device Model for Polymeric Light Emitting Diodes. J. Phys. D: Appl. Phys. 32 (1999) 2973-2984

14. Kawabe, Y., Jabbour, G. E., Shaheen, S. E., Kippelen, B., Peyghambarian, N.: A Model for the Current-Voltage Characteristics and the Quantum Efficiency of Single-Layer Organic Light Emitting Diodes. Appl. Phys. Lett. 71 (1997) 1290-1292 